ARTICLE

\title{
Force generation by a propagating wave of supramolecular nanofibers
}

\author{
Ryou Kubota (i) ${ }^{1}$, Masahiro Makuta², Ryo Suzuki (i) ${ }^{3}$, Masatoshi Ichikawa (i) ${ }^{2}$, Motomu Tanaka (i]) ${ }^{3,4}$ \& \\ Itaru Hamachi (1D) 1,5凶
}

Dynamic spatiotemporal patterns that arise from out-of-equilibrium biochemical reactions generate forces in living cells. Despite considerable recent efforts, rational design of spatiotemporal patterns in artificial molecular systems remains at an early stage of development. Here, we describe force generation by a propagating wave of supramolecular nanofibers. Inspired by actin dynamics, a reaction network is designed to control the formation and degradation of nanofibers by two chemically orthogonal stimuli. Real-time fluorescent imaging successfully visualizes the propagating wave based on spatiotemporally coupled generation and collapse of nanofibers. Numerical simulation indicates that the concentration gradient of degradation stimulus and the smaller diffusion coefficient of the nanofiber are critical for wave emergence. Moreover, the force $(0.005 \mathrm{pN})$ generated by chemophoresis and/or depletion force of this propagating wave can move nanobeads along the wave direction.

\footnotetext{
${ }^{1}$ Department of Synthetic Chemistry and Biological Chemistry, Graduate School of Engineering, Kyoto University, Katsura, Nishikyo-ku, Kyoto 615-8510, Japan. ${ }^{2}$ Department of Physics, Kyoto University, Sakyo-ku, Kyoto 606-8502, Japan. ${ }^{3}$ Center for Integrative Medicine and Physics, Institute for Advanced Study, Kyoto University, Sakyo-ku, Kyoto 606-8501, Japan. ${ }^{4}$ Physical Chemistry of Biosystems, Institute of Physical Chemistry, Heidelberg University, 69120 Heidelberg, Germany. ${ }^{5}$ JST-ERATO, Hamachi Innovative Molecular Technology for Neuroscience, Kyoto University, Katsura, Nishikyo-ku, Kyoto 615-8530,

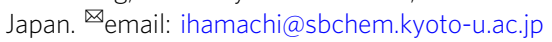


O ut-of-equilibrium events in chemistry and biology are abundant in nature at all scales, providing a variety of spatial-temporal patterns, such as oscillations, waves, and spirals $^{1-4}$. In living cells, dynamic spatiotemporal patterns play essential roles in cellular functions, such as Min systems for division and actin waves for migration ${ }^{5-7}$. Force is also produced by such spatiotemporal patterns ${ }^{5,6}$. In lamellipodia, the propagating wave of actin filaments can generate the force required to push the cell membrane forward for cell migration, as has been well studied by single-molecule force spectroscopy ${ }^{8-11}$.

Inspired by these cellular systems, out-of-equilibrium dynamic patterns of supramolecular architectures are anticipated to give lifelike characteristics, such as autonomy, adaptivity, and homeostasis, to artificial soft materials ${ }^{12-38}$. For example, polymer-based soft materials coupled with the Belousov-Zhabotinsky reaction can exhibit sophisticated functions such as self-oscillation of gels, selfwalking actuators, and autonomous mass transport ${ }^{39-42}$. Recently, formation of spatiotemporal patterns relying on stimulusresponsive supramolecular nanofibers has been proposed. A fueldriven dissipative supramolecular system, pioneered by van Esch et al., showed stochastic formation and degradation of supramolecular nanofibers, albeit not coupled in time and space ${ }^{22}$. Hermans et al. reported a traveling front of supramolecular colloids in a nonstirred reaction vessel; however, the degradation did not occur simultaneously $y^{31}$. Despite considerable efforts, rational guidelines have yet to be determined for generating spatiotemporal patterns based on formation and degradation of supramolecular nanofibers. Moreover, precise measurements of the forces generated by artificial spatiotemporal patterns have yet to be achieved. There have been many examples of force generation by out-of-equilibrium systems in biology; however, it is reasonable to expect that artificial spatiotemporal patterns of supramolecular nanofibers can also generate forces. Such efforts might bridge biological and artificial systems, which is a crucial step towards design of next-generation soft materials with cell-mimetic functions.

We herein describe force generation by a propagating wave of supramolecular nanofibers comprising a peptide-type hydrogelator (Fig. 1a). In lamellipodia of cell migration, distinct accessory proteins such as Arp $2 / 3$ complex and ADF/cofilin independently control the kinetics of actin polymerization and depolymerization in a spatiotemporal manner ${ }^{5,6}$. Inspired by actin dynamics, we design a reaction network to control the formation and degradation of supramolecular nanofibers comprising a short peptide by two chemically orthogonal stimuli. Real-time confocal laser scanning microscopic (CLSM) imaging visualizes that the propagating wave based on spatiotemporally coupled generation and collapse of nanofibers proceeds in the $\mathrm{mm}$ scale. Numerical simulation and analysis indicate that the concentration gradients of the formation and degradation stimuli are one of the main controlling factors and the smaller diffusion coefficient of the nanofiber is critical for wave emergence. Moreover, we succeed in quantitative determination of the force generated by this propagating wave, which can move nanobeads along the wave direction.

\section{Results}

Design of a chemical reaction network for a propagating wave. To realize the supramolecular propagation wave by imitating dynamic actin assembly/disassembly, we set the following four guidelines to design a chemical reaction network for an artificial propagating wave. First, the propagating wave is driven by a concentration gradient of chemical stimuli. Second, two distinct chemical stimuli can induce formation or degradation of the nanofibers. Third, a monomer has two different functional groups, each of which orthogonally responds to one distinct stimulus. a

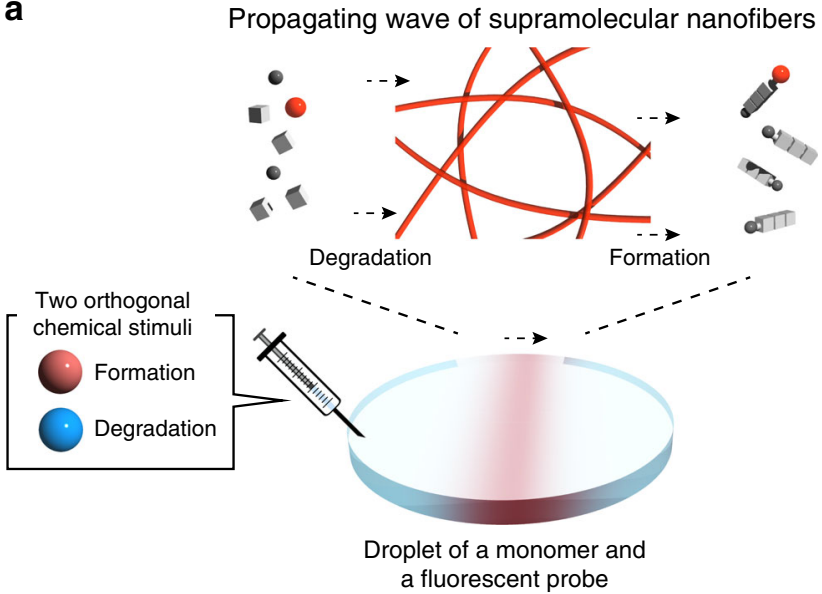

b

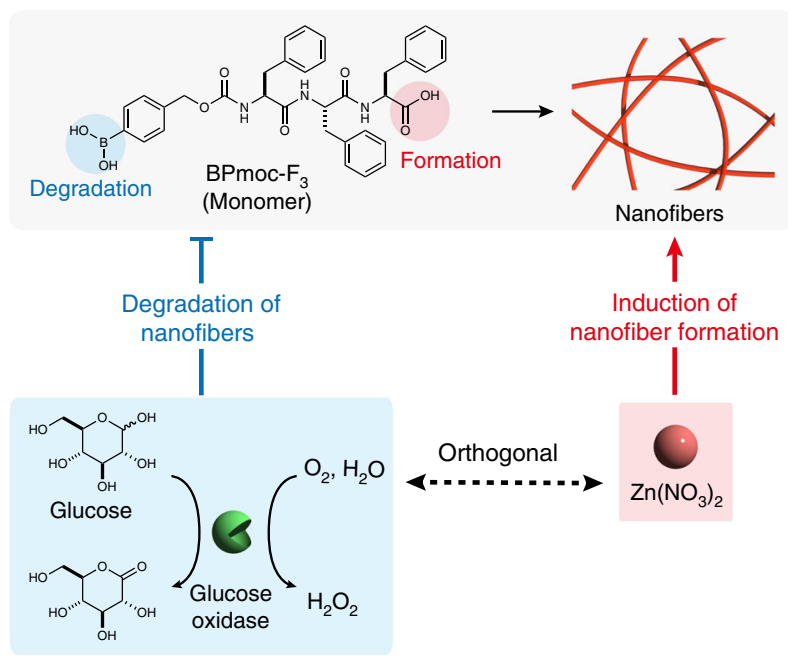

C

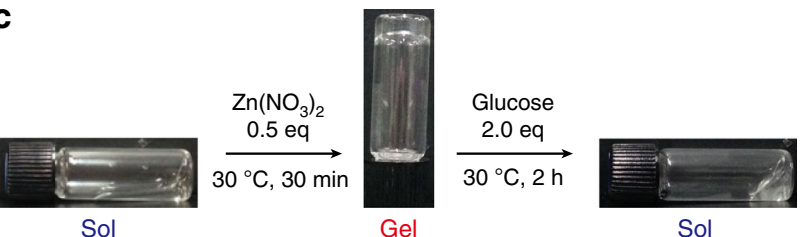

Fig. 1 Design of a propagating wave of supramolecular nanofibers. a Schematic illustration of a propagating wave comprising spatiotemporally coupled formation and degradation of supramolecular nanofibers driven by the concentration gradient of two chemically orthogonal stimuli. b A reaction network for the supramolecular propagating wave. $\mathrm{Zn}\left(\mathrm{NO}_{3}\right)_{2}$ induces the nanofiber formation, while $\mathrm{H}_{2} \mathrm{O}_{2}$ produced by enzymatic reaction of glucose oxidase with glucose collapses the nanofibers. The formation and degradation stimuli are chemically orthogonal to each other. c Macroscopic sol-gel-sol transition of $\mathrm{BPmoc}-\mathrm{F}_{3}$ containing glucose oxidase (GOx) upon addition of $\mathrm{Zn}\left(\mathrm{NO}_{3}\right)_{2}(0.5 \mathrm{eq})$ and glucose $(2.0 \mathrm{eq})$. Condition: $\left[\mathrm{BPmoc}-\mathrm{F}_{3}\right]=2.4 \mathrm{mM}$, $[\mathrm{GOx}]=1 \mathrm{mg} / \mathrm{mL},\left[\mathrm{Zn}\left(\mathrm{NO}_{3}\right)_{2}\right]=1.2 \mathrm{mM}$, [glucose $]=4.8 \mathrm{mM}, 50 \mathrm{mM}$ HEPES, pH 7.4.

Fourth, the two stimuli should not interfere with each other to enable precise control of the reaction conditions. Here, we selected BPmoc- $\mathrm{F}_{3}$ hydrogelator as a monomer (Fig. 1b). BPmoc- $\mathrm{F}_{3}$ has two functional groups for formation and degradation of supramolecular nanofibers: carboxylate and boronobenzyl groups at the C- and Ntermini, respectively. $B P m o c-F_{3}$ forms supramolecular nanofibers in an aqueous buffer solution, and the BPmoc- $\mathrm{F}_{3}$ nanofibers decompose upon treatment of oxidase/substrate pairs (e.g., glucose oxidase (GOx) and glucose) through a 1,6-elimination reaction on the 
boronobenzyl oxycarbonyl group by $\mathrm{H}_{2} \mathrm{O}_{2}$ (Fig. 1 b, Supplementary Fig. 1 $)^{43,44}$. For the formation stimulus we used $\mathrm{Zn}^{2+}$ ions because the carboxylate group acts as a coordination site to trigger nanofiber formation by bridging $\mathrm{BPmoc}-\mathrm{F}_{3}$ monomers and/or suppressing anionic charge repulsion. Furthermore, the $\mathrm{Zn}^{2+}$ ion is redox-inert so that it will not interfere with glucose/GOx stimulus (generating $\left.\mathrm{H}_{2} \mathrm{O}_{2}\right)^{45}$. Therefore, it is conceivable that the $\mathrm{Zn}^{2+}$ ion and GOx/ glucose are orthogonal stimuli that might induce formation and decomposition of $\mathrm{BPmoc}-\mathrm{F}_{3}$ nanofibers, respectively.

Stimulus-responsive nanofiber formation and degradation. We initially investigated $\mathrm{Zn}^{2+}$ ion-triggered hydrogelation of BPmoc$\mathrm{F}_{3}$ by a tube inversion method. After addition of 0.5 eq of $\mathrm{Zn}^{2+}$ ion, an aqueous HEPES buffer solution of BPmoc- $\mathrm{F}_{3}$ changed into a transparent hydrogel within $30 \mathrm{~min}$ (Fig. 1c, Supplementary Fig. 2a). The resulting hydrogel turned back to a sol state upon addition of 0.5 eq of EDTA. We also confirmed that neither a sol-gel nor a gel-sol transition took place upon addition of $\mathrm{H}_{2} \mathrm{O}$ or buffer instead of $\mathrm{Zn}\left(\mathrm{NO}_{3}\right)_{2}$ or EDTA solution, respectively (Supplementary Fig. 2b, c). These results indicated the potential for coordination-driven hydrogelation of $\mathrm{BPmoc}-\mathrm{F}_{3}$. We next examined the responsiveness of the $\mathrm{Zn}^{2+}$-induced $\mathrm{BPmoc}-\mathrm{F}_{3}$ nanofibers towards a pair of GOx and glucose. The $\mathrm{Zn}^{2+}$-induced hydrogel containing GOx changed into the solution state after incubation at $30^{\circ} \mathrm{C}$ for $2 \mathrm{~h}$ in the presence of glucose $(2.0 \mathrm{eq})$ (Fig. 1c). As a control, the hydrogel maintained the gel state upon addition of the same amount of $\mathrm{H}_{2} \mathrm{O}$ (Supplementary Fig. 3a). HPLC analyses after glucose treatment demonstrated that $\mathrm{BPmoc}-\mathrm{F}_{3}$ was completely decomposed within $2 \mathrm{~h}$, whereas over $97 \%$ of BPmoc- $\mathrm{F}_{3}$ remained upon $\mathrm{H}_{2} \mathrm{O}$ treatment (Supplementary Fig. 3b, c).

We then performed real-time CLSM imaging of the formation and degradation of $\mathrm{Zn}^{2+}$-induced BPmoc- $\mathrm{F}_{3}$ nanofibers stained with BP-TMR, a fluorescent probe (Supplementary Fig. 4). After addition of $\mathrm{Zn}\left(\mathrm{NO}_{3}\right)_{2}$ (0.5 eq) to a homogeneous solution of BPmoc- $\mathrm{F}_{3}$ and BP-TMR in HEPES buffer, thin fibrous nanofibers gradually formed (Supplementary Fig. 5a, Supplementary Movie 1). Observation of the formation process showed that aggregate-like seeds stochastically formed, and then nanofibers grew from the seeds to connect the different seeds/fibers, resulting in the formation of a fiber network (Supplementary Fig. 5b). The fluorescence intensity profile of the CLSM images revealed that the nanofiber formation continued for over $60 \mathrm{~min}$ (Supplementary Fig. 5c). Furthermore, real-time CLSM imaging allowed us to visualize the fiber degradation process upon addition of glucose $(2.0 \mathrm{eq})$ to the hydrogel containing GOx (Supplementary Figs. 6a and 7, Supplementary Movie 2). A time-course plot of the fluorescent intensity changes showed that $\mathrm{Zn}^{2+}$-induced nanofibers homogeneously disappeared over $60 \mathrm{~min}$ (Supplementary Fig. 6c). We also confirmed that the interaction between $\mathrm{Zn}\left(\mathrm{NO}_{3}\right)_{2}$ and the GOx/glucose pair was negligible in nanofiber formation and degradation processes (Supplementary Figs. 8 and 9).

Real-time imaging of a supramolecular propagating wave. With formation and degradation stimuli of the supramolecular nanofibers in hand, we attempted to generate the propagating wave of supramolecular nanofibers upon simultaneous treatment of $\mathrm{Zn}\left(\mathrm{NO}_{3}\right)_{2}$ and glucose. To observe the propagating wave by real-time CLSM imaging, we prepared a droplet containing BPmoc- $F_{3}, B P-T M R$, and GOx, which was sandwiched between two glass plates (Fig. 2a). Time-lapse CLSM imaging after addition of a mixture of $\mathrm{Zn}\left(\mathrm{NO}_{3}\right)_{2}(0.5 \mathrm{eq})$ and glucose $(2.0 \mathrm{eq})$ via a syringe at the right edge of the droplet clearly visualized the generation of the propagating wave based on spatially-coupled formation and degradation of supramolecular nanofibers (Fig. 2b,
Supplementary Movie 3). Immediately after addition of $\mathrm{Zn}\left(\mathrm{NO}_{3}\right)_{2}$ and glucose, small seeds stochastically formed. Thereafter (around $10 \mathrm{~min}$ ), the nanofibers gradually appeared from the right side and grew up towards the left side, followed by degradation of the supramolecular nanofibers along the same direction. After the wave propagation, no notable fluorescence was observed, suggesting that the BPmoc- $\mathrm{F}_{3}$ fibers were completely decomposed. The time course of the fluorescent intensity at distinct $x$ coordinates quantitatively demonstrated that the time of the maximum fluorescent intensity was delayed as the $x$ coordinate increased (Fig. 2c). Hence, the supramolecular wave clearly propagated along the $x$ axis. In contrast to simultaneous growth and shrinkage of nanofibers in proximity reported by van Esch $^{22}$, nanofiber formation and degradation in this propagating wave were highly regulated in a spatiotemporal manner by the concentration gradient of stimuli, which would suppress the stochastic nature of the fiber formation/degradation process.

To investigate the behavior of the propagating wave in detail, we next conducted CLSM imaging over a wider field of view (Fig. 2e, Supplementary Movie 4). After addition of a mixture of $\mathrm{Zn}\left(\mathrm{NO}_{3}\right)_{2}$ ions and glucose, a crescent-shaped bright area immediately appeared. After $15 \mathrm{~min}$, the fluorescence at the right edge of the crescent region became brighter. The increase of the fluorescent intensity propagated to the left, whereas the fluorescent intensity at the original position decreased. To quantitatively assess the behavior of the supramolecular propagating wave, we plotted the time course of the fluorescent intensity changes at various distances from the original (Fig. 2f). As a typical example, the fluorescent intensity at $x=1.3 \mathrm{~mm}$ gradually increased during the initial $5 \mathrm{~min}$, likely because of nanofiber formation (Fig. 2f, red line). After $25 \mathrm{~min}$, the fluorescent intensity increased nonlinearly and reached its maximum at $30 \mathrm{~min}$. Then, the fluorescent intensity decreased for the next $5 \mathrm{~min}$. The time course plot reveals that a propagating wave was generated in the macroscopic range ( $\mathrm{mm}$ scale). The wave started at $x=1.1 \mathrm{~mm}$ and completely disappeared at $x=1.5 \mathrm{~mm}$. On the basis of these observations, the propagating distance and the average velocity of the propagating wave were estimated to be $340 \pm 40 \mu \mathrm{m}$ and $54 \pm 8 \mu \mathrm{m} / \mathrm{min}$, respectively (Supplementary Fig. 10).

The formation of the supramolecular propagating wave was strongly dependent on the reaction conditions. When half concentration of GOx was used $(0.5 \mathrm{mg} / \mathrm{mL})$, the propagating wave was indeed formed, while the duration of the propagating wave slightly increased and the velocity decreased to be $20 \pm$ $2 \mu \mathrm{m} / \mathrm{min}$ (Supplementary Fig. 11, Supplementary Movie 5). On the other hand, a propagating wave was not observed when a smaller amount of glucose (1.0 eq) was used (Supplementary Fig. 12a, Supplementary Movie 6). CLSM imaging showed that the nanofibers were indeed formed over 60 min immediately after addition of the two stimuli; however, the system degraded homogenously. The time course change of the fluorescent intensity was independent of the $x$-coordinates, suggesting that the propagating wave did not form under these conditions (Supplementary Fig. 12b). When a larger amount of GOx $(2 \mathrm{mg} / \mathrm{mL})$ or glucose $(63 \mathrm{eq})$ was used, we observed negligible formation of nanofibers owing to rapid degradation of BPmoc$\mathrm{F}_{3}$ (Supplementary Figs. 13 and 14). To confirm whether simultaneous addition of $\mathrm{Zn}\left(\mathrm{NO}_{3}\right)_{2}$ and glucose is important for the wave formation, we treated the droplets of BPmoc- $\mathrm{F}_{3}$, BP-TMR, and GOx by addition of glucose 30 min after $\mathrm{Zn}$ $\left(\mathrm{NO}_{3}\right)_{2}$ injection (nanofiber formation is saturated after incubation for $30 \mathrm{~min}$ as shown in Supplementary Fig. 5). The BPmoc- $\mathrm{F}_{3}$ nanofibers formed upon treatment with $\mathrm{Zn}$ $\left(\mathrm{NO}_{3}\right)_{2}$ and begun to homogenously disappear $50 \mathrm{~min}$ after addition of glucose, indicating no generation of a propagating wave (Fig. 2d, Supplementary Fig. 15, Supplementary Movie 7). 
a

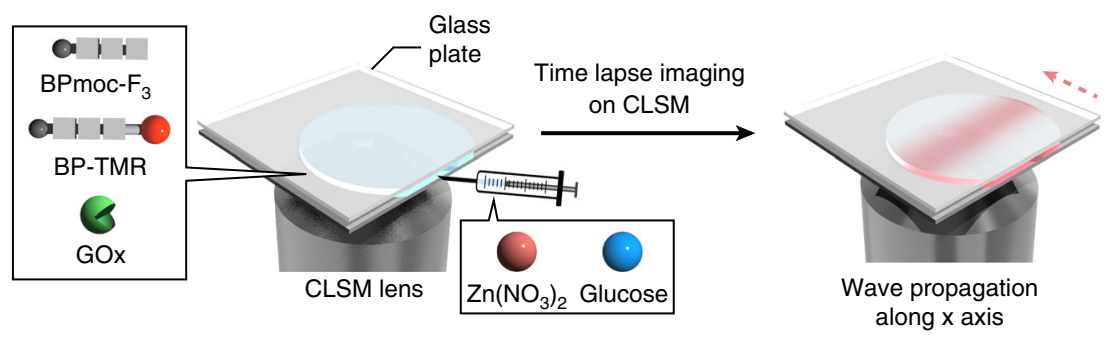

b Injection was conducted from the right side of images
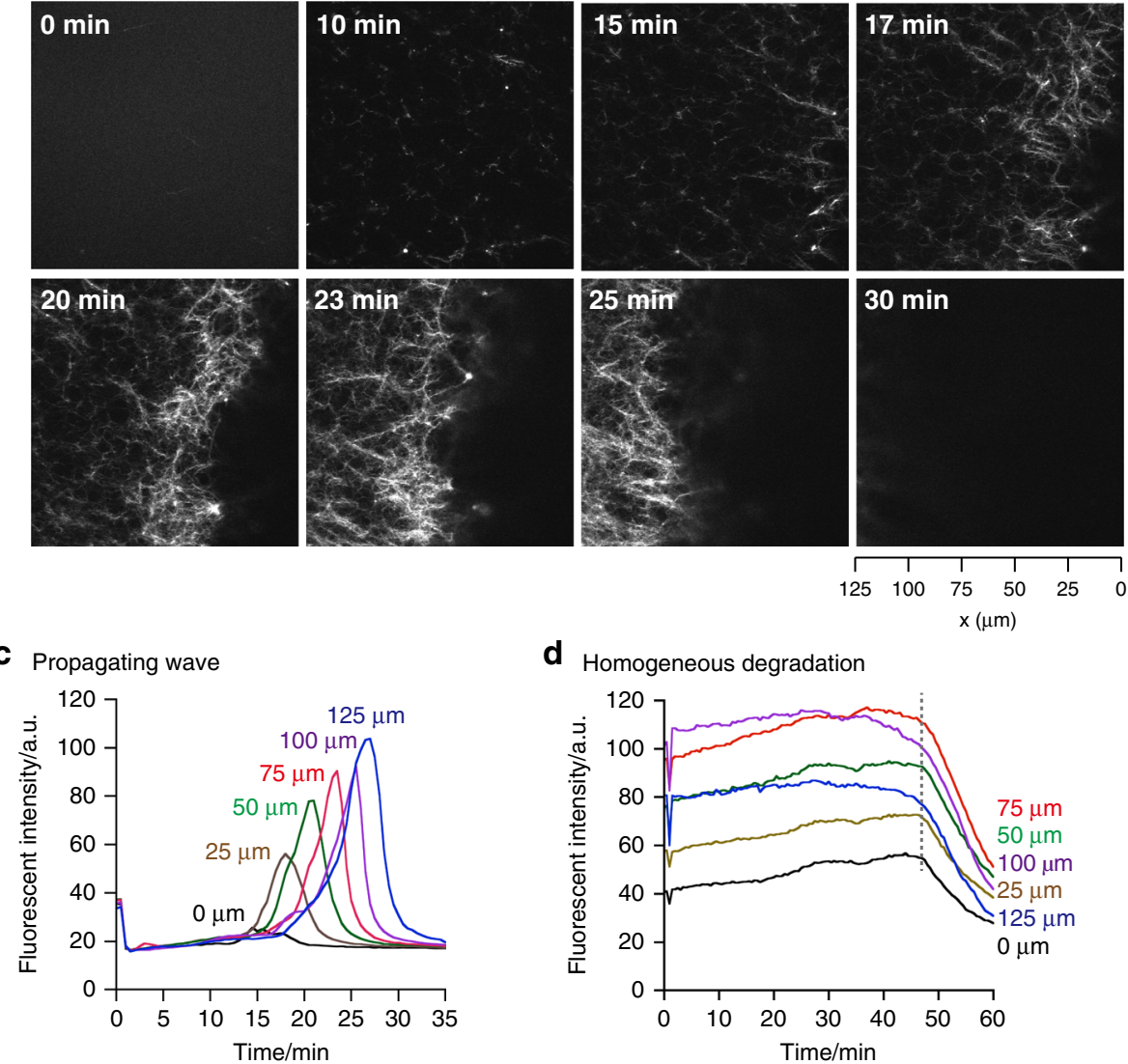

d

Homogeneous degradation

e
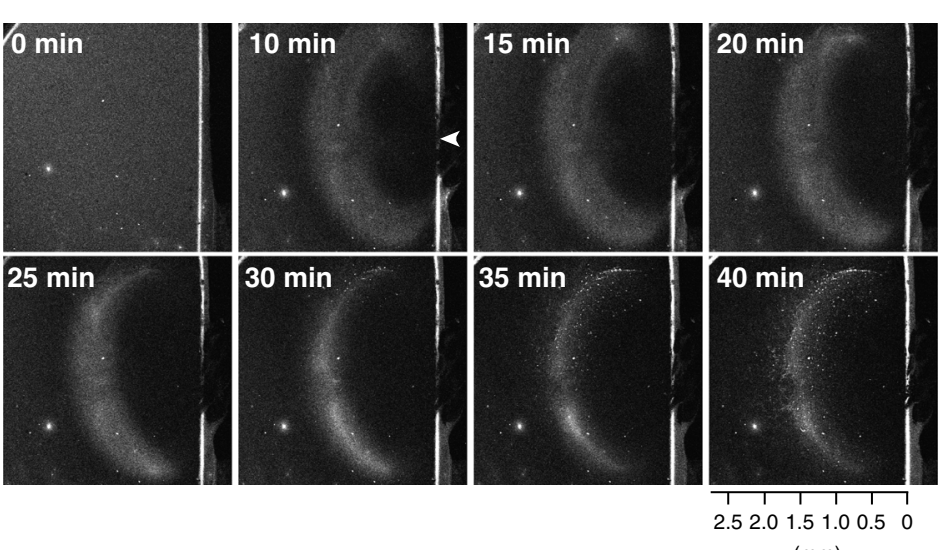

$\mathbf{f}$

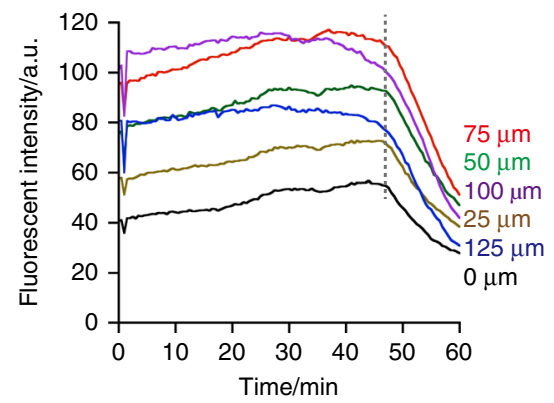

It implies that concentration gradient of $\mathrm{Zn}\left(\mathrm{NO}_{3}\right)_{2}$ would play an important role for wave formation. We also confirmed that pretreatment of the glucose before addition of $\mathrm{Zn}^{2+}$ ions did not induce nanofiber formation (Supplementary Fig. 16). These observations indicated that simultaneous addition of $\mathrm{Zn}^{2+}$ and glucose at a critical concentration would kinetically balance the formation/degradation of the supramolecular fibers, which is crucial for the generation of the propagating wave.

Numerical simulation of the supramolecular propagating wave. To gain further insights into the supramolecular propagating 
Fig. 2 In situ real-time imaging of a propagating wave of supramolecular nanofibers. a Schematic illustration of the experimental setup. A solution of BPmoc- $\mathrm{F}_{3}, \mathrm{BP}-\mathrm{TMR}$, and GOx was placed between two glass plates. One minute after starting confocal laser scanning microscopic (CLSM) observation, two stimuli were added via a syringe from the right edge of the droplet. $\mathbf{b}$, e Time-lapse CLSM imaging of the propagating wave of supramolecular nanofibers observed by $\mathbf{b} 100 \times$ and $\mathbf{e} 4 \times$ objectives. $\mathbf{b}$ A solution of $\mathrm{Zn}\left(\mathrm{NO}_{3}\right)_{2}$ and glucose was injected from the right side of images, and $\mathbf{e}$ the injection point was highlighted by an arrowhead. $\mathbf{c}, \mathbf{d}, \mathbf{f}$ Time-course of fluorescent intensity at distinct $x$-coordinates under the propagating wave (c Fig. $2 b$ and $\mathbf{f} 2 \mathrm{e}$ ) and $\mathbf{d}$ homogeneous nanofiber degradation (Supplementary Fig. 15). The shape of the crescent-shaped area varied depending on experiments because injection of the chemical stimuli was conducted manually; nevertheless the formation of the propagating wave is highly reproducible (Supplementary Fig. 10). Condition: $\left[B P m o c-F_{3}\right]=1.6 \mathrm{mM},[B P-T M R]=0.34 \mu \mathrm{M},[\mathrm{GOx}]=1.0 \mathrm{mg} / \mathrm{mL},\left[\mathrm{Zn}\left(\mathrm{NO}_{3}\right)_{2}\right]=0.8 \mathrm{mM}$, [glucose $]=3.2 \mathrm{mM}$ in $50 \mathrm{mM} \mathrm{HEPES}, \mathrm{pH}$ $7.4,30^{\circ} \mathrm{C}$.

wave, we performed a numerical simulation based on a reactiondiffusion model. Because of the formation processes of the supramolecular nanofibers, we supposed three reaction steps: (i) $\mathrm{Zn}^{2+}$-promoted nanofiber formation between monomers, (ii) $\mathrm{Zn}^{2+}$-dependent and (iii) $\mathrm{Zn}^{2+}$-independent nanofiber elongation processes. These formation processes are linearized. The decomposition of the monomer and nanofibers were assumed to be a second-order reaction based on the degradation stimulus. The interference between the formation and degradation stimuli was ignored owing to their chemical orthogonality. The concentrations of the stimuli were decreased only by molecular diffusion because the formation stimulus should be reused and an excess amount of degradation stimulus was added. From these assumptions, we obtained a set of reaction-diffusion equations:

$$
\begin{aligned}
& \frac{\partial n}{\partial t}=D_{n} \nabla^{2} n+k_{1} m x+k_{2} n m-k_{3} n y+k_{5} n m x \\
& \frac{\partial m}{\partial t}=D_{m} \nabla^{2} m-k_{1} m x-k_{2} n m-k_{4} m y \\
& \frac{\partial x}{\partial t}=D_{x} \nabla^{2} x \\
& \frac{\partial y}{\partial t}=D_{y} \nabla^{2} y
\end{aligned}
$$

where, $n, m, x$, and $y$ are defined as concentrations of the nanofibers, monomer, formation, and degradation stimuli, respectively. For the numerical simulation, we considered that the $k_{2}$ value and the diffusion coefficient of the nanofibers should be much smaller than other kinetic and diffusion coefficients. Based on these equations, we successfully reproduced the propagating wave under the appropriate conditions, as shown in Fig. 3a and Supplementary Movie 8. The numerical simulation demonstrates that a band of supramolecular nanofibers with limited width proceeded to the left, as shown in the kymograph (Fig. 3b). In addition, the monomer had a spatial concentration gradient, which strongly depended on the concentration gradient of the formation and degradation stimuli. This result suggests that these concentration gradients were one of the main driving forces for wave formation. Numerical analysis also provided other important insights into the propagating wave; the wave velocity was proportional to the formation kinetics of the nanofibers and the diffusion coefficient of the degradation stimulus, and the initial anisotropic shapes of the formation and degradation stimuli rapidly decayed into half-round distributions and were not crucial to the crescent-shaped propagation. In addition, it is implied that the concentration gradient of the degradation stimulus is a key parameter for maintaining wave propagation (see SI for details). Furthermore, the much smaller diffusion coefficient of the nanofibers is another important factor for producing a propagating wave, suggesting that a supramolecular nanofiber is one of the most suitable scaffolds for spatiotemporal pattern formation. The numerical simulation also suggested that the small
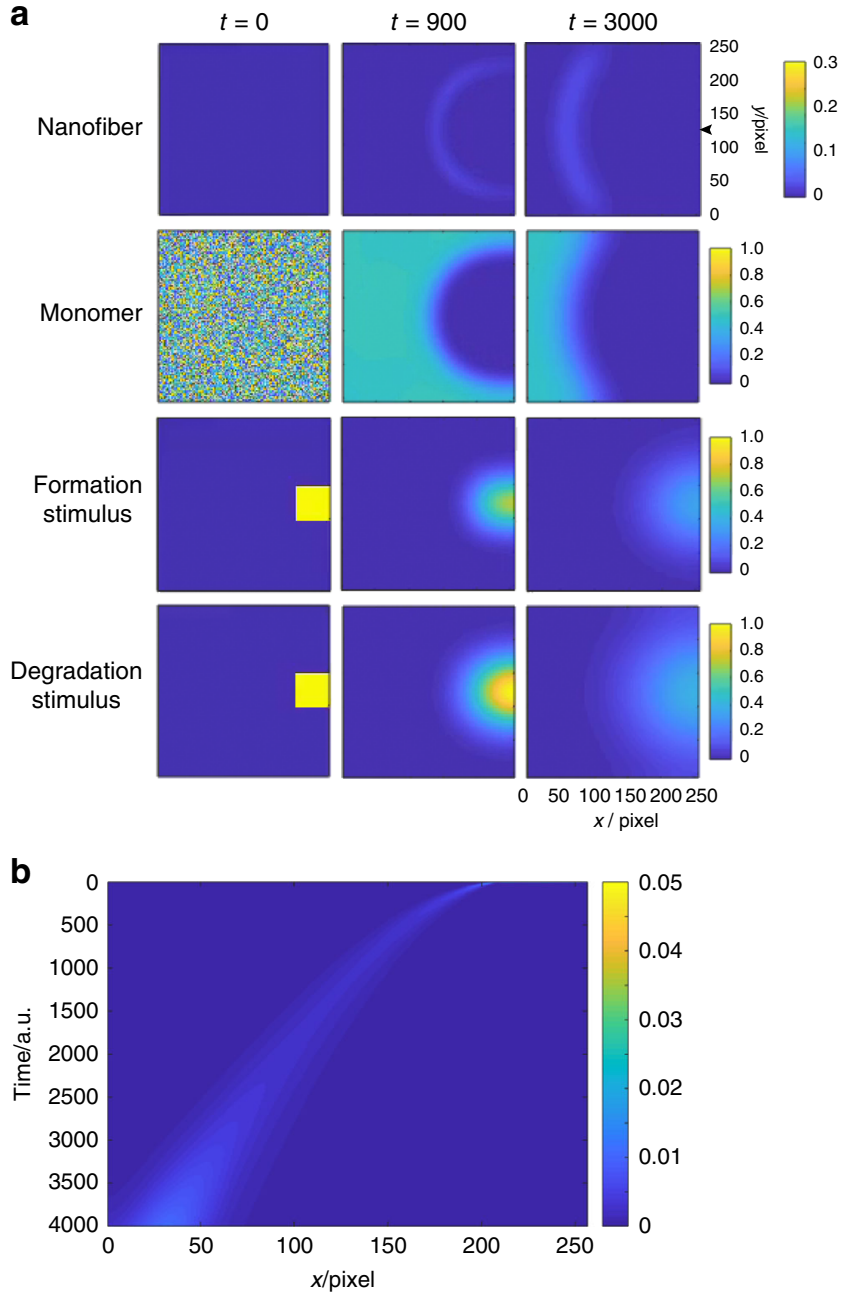

Fig. 3 Numerical simulation of the propagating wave based on a reactiondiffusion mechanism. a Time dependent concentration changes of (top) supramolecular nanofibers, (2nd row) monomer, (3rd row) formation, and (bottom) degradation stimuli. Please see the main text and SI for the reaction-diffusion equations. $\mathbf{b}$ Kymograph of the nanofiber concentration on the center at $y$ axis ( $y=128$, highlighted by a black arrowhead) of Fig. 3a. Condition: $D_{\mathrm{n}}: 0.0001, D_{\mathrm{m}}: 0.003, D_{\mathrm{x}}: 0.003, D_{\mathrm{y}}: 0.006, k_{1}: 0.1, k_{2}$ : $0.01, k_{3}: 0.4, k_{4}: 0.2, k_{5}: 0.1$

perturbation of diffusion (by microfluidics, for example) would enable the modulation of wave propagation. Our numerical simulation demonstrates that the design principle of the reaction network based on orthogonal formation and degradation stimuli is a promising strategy for forming supramolecular propagating waves. 
a

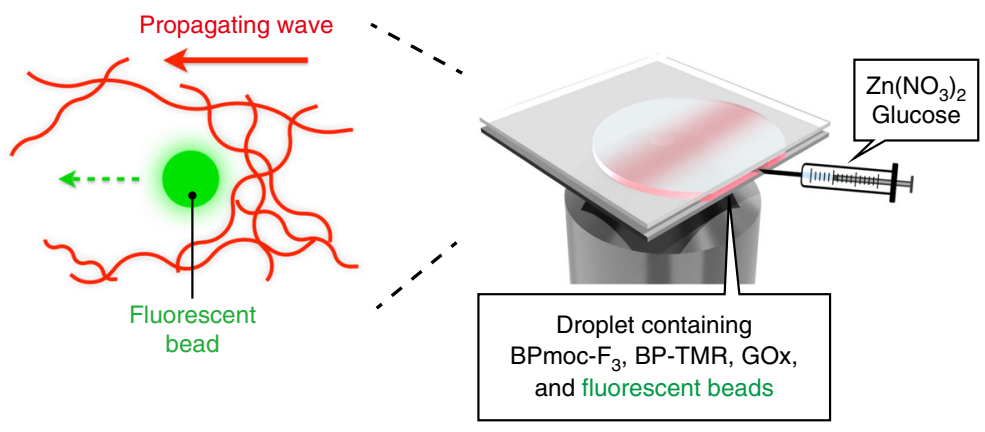

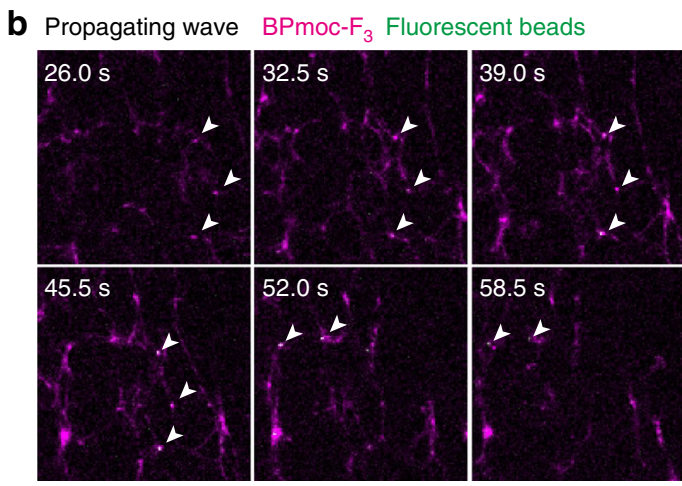

d Homogeneous degradation

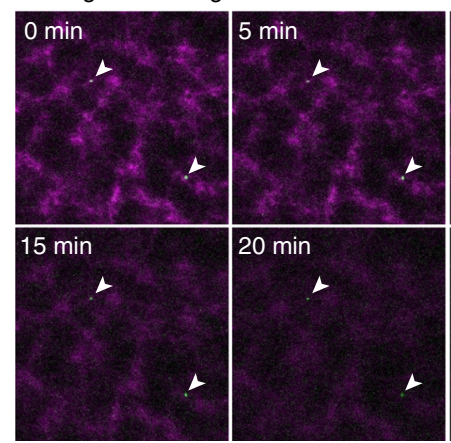

$\overline{20 \mu \mathrm{m}}$

C

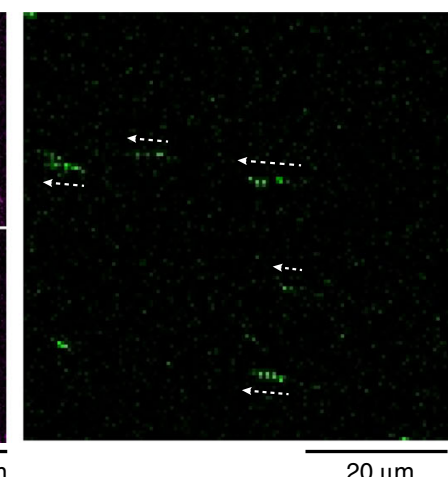

e

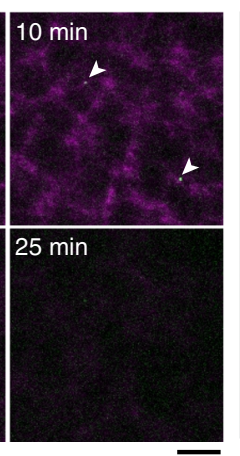

$\overline{20 \mu \mathrm{m}}$

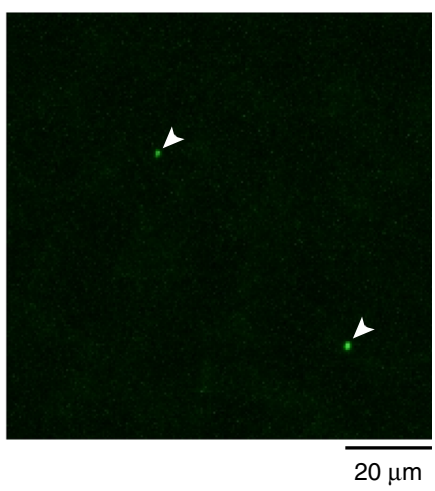

Fig. 4 Force generation by the supramolecular propagating wave. a Schematic illustration of the bead movement induced by the propagating wave. $\mathbf{b}, \mathbf{d}$ Time-lapse images of the bead displacement under $\mathbf{b}$ the propagating wave and $\mathbf{d}$ homogeneous degradation of supramolecular nanofibers. Time at $\mathbf{b}$ starting the time-lapse imaging or $\mathbf{d}$ adding glucose was defined as $0 . \mathbf{c}, \mathbf{e}$ Trajectories of the intensity maxima from the fluorescently labeled beads under c the propagating wave and $\mathbf{e}$ homogeneous nanofiber degradation monitored over $\mathbf{c} 26 \mathrm{~s}$ and $\mathbf{e} 25$ min, respectively. White arrowheads show the position of fluorescently labeled beads. Green: fluorescently labeled beads, magenta: BPmoc- $F_{3} / B P-T M R$. As shown in Supplementary Fig. 17, time course of fluorescent intensity of nanofibers showed that the propagating wave formed in Fig. 4b. Time-lapse CLSM images of the bead displacement under homogeneous nanofiber formation were shown in Supplementary Fig. 18. Condition: $\left[B P m o c-F_{3}\right]=1.6 \mathrm{mM},[B P-T M R]=0.34 \mu M,[G O x]=1.0 \mathrm{mg} / \mathrm{mL}$, $\left[\mathrm{Zn}\left(\mathrm{NO}_{3}\right)_{2}\right]=0.8 \mathrm{mM}$, [beads $]=20 \mu \mathrm{g} / \mathrm{mL}$, [glucose $]=3.2 \mathrm{mM}$ in $50 \mathrm{mM} \mathrm{HEPES}, \mathrm{pH} 7.4,30^{\circ} \mathrm{C}$.

Force generation by the supramolecular propagating wave. We finally attempted to examine if a force is generated by this supramolecular propagating wave. The experiment and simulation predicted that the propagating wave should generate the forces that depends on the gradient of chemical (chemophoresis), and that depends on the concentration gradient of the fibrous supramolecules which depletes larger objects from the higher concentrated region (depletion or entropy effects). To evaluate the force quantitatively, we monitored the displacement of PEGcoated fluorescently labeled polystyrene beads (diameter: $500 \mathrm{~nm}$ ) during the wave propagation (Fig. 4a). Real-time CLSM imaging clearly indicated that the beads move along the direction of the propagating wave. As shown in Fig. 4b, c, and Supplementary Movie 9, multiple beads appeared in the field of view at the beginning of the wave formation and continued to move during the propagation, resulting in a displacement of $c a .10 \mu \mathrm{m}$. The time-profile of the trajectory revealed the average velocity of the beads was $c a .0 .4 \mu \mathrm{m} / \mathrm{s}$. Taking the viscosity of the medium to be ca. $2.5 \mathrm{mPa} \cdot \mathrm{s}$, the minimal force generated by the propagation wave was calculated by the Stokes law to be $c a .0 .005 \mathrm{pN}$ (see Methods for the detailed calculation). As a control, we confirmed that the homogeneous nanofiber formation and degradation did not cause notable movement of the beads (Fig. 4d, e, Supplementary Fig. 18, Supplementary Movies 10 and 11). To the best of our knowledge, this is the first example of quantitative determination of the force generated by the spatiotemporal pattern of artificial supramolecular systems. Compared with the biological examples [the stalling force of actin and microtubule 
polymerization (0.76 and $2.7 \mathrm{pN}$ per single filament, respectively)], the force generated by our supramolecular propagating wave is rather small ${ }^{9,11}$. To examine if our fibers are soft and undergo bending deformation, we measured the persistence length of the supramolecular nanofiber based on thermal fluctuation (Supplementary Fig. 19, Supplementary Movie 12) ${ }^{46}$. The persistence length of our nanofibers $\left(\xi_{\text {fiber }} \approx 25 \mu \mathrm{m}\right)$ is comparable to that of actin filaments $\left(\xi_{\text {actin }} \approx 18 \mu \mathrm{m}\right)^{47}$. Because our current supramolecular wave consists of physically entangled nanofibers, more precise (vectorial) control of the supramolecular nanofiber structures (e.g., crosslinked, bundled, and branched networks ${ }^{48}$ ) will enable us to cover a range of forces comparable to those in biological systems ( $\mathrm{pN}$ to $\mathrm{nN}$ order) ${ }^{10}$. Such behaviors have not been realized by dissipative synthetic assembly to date.

\section{Methods}

General. Unless stated otherwise, all commercial reagents were used as received. Thin-layer chromatography (TLC) was performed on silica gel $60 \mathrm{~F}_{254}$ (Merck). ${ }^{1} \mathrm{H}$ NMR spectra were obtained on a Varian Mercury 400 spectrometer with residual non-deuterated solvents $\left(\mathrm{CD}_{3} \mathrm{OD}: 3.31 \mathrm{ppm}\right.$ for $\left.{ }^{1} \mathrm{H}\right)$ as the internal reference. ESI mass spectra were recorded using an Exactive (Thermo Scientific). Reversed-phase HPLC (RP-HPLC) was carried out on a Hitachi Chromaster system equipped with a diode array and YMC-Pack Triart C18 or ODS-A columns. All runs used linear gradients of acetonitrile (ACN) containing $0.1 \%$ trifluoroacetic acid (TFA) and $0.1 \%$ aqueous TFA. The images of confocal laser scanning microscopy (CLSM) were acquired by a FV1000 (Olympus) and a LSM800 (Carl Zeiss Microscopy). UPlanSApo $100 \times$ (1.40 numerical aperture, oil immersion, Olympus), UPlanSApo $4 \times$ (0.16 numerical aperture, Olympus), Plan-Apochromat $20 \times(0.8$ numerical aperture, Carl Zeiss), and aPlan-Apochromat 100× (1.46 numerical aperture, Carl Zeiss) were used. The fluorescent intensity of the CLSM images was calculated by Fiji ${ }^{4}$.

Preparation of a homogeneous BPmoc- $\mathbf{F}_{3}$ solution. A BPmoc- $\mathrm{F}_{3}$ powder was suspended in an aqueous buffer (50 mM HEPES, $\mathrm{pH}$ 7.4), and heated until dissolving. After cooling to room temperature, 10X stock solutions of BP-TMR (3.4 $\mu \mathrm{M}$ in $50 \mathrm{mM}$ HEPES, $\mathrm{pH} 7.4)$ and/or GOx $(10 \mathrm{mg} / \mathrm{mL}$ in $50 \mathrm{mM}$ HEPES, $\mathrm{pH} 7.4$ containing $5 \%(\mathrm{v} / \mathrm{v})$ glycerol) were added.

$\mathbf{Z n}^{\mathbf{2}+}$ ion-induced gelation of BPmoc- $\mathbf{F}_{3}$. To a freshly-prepared aqueous solution of BPmoc- $\mathrm{F}_{3}(2.4 \mathrm{mM}, 0.15 \mathrm{wt} \%, 50 \mathrm{mM}$ HEPES buffer (pH 7.4), $100 \mu \mathrm{L}$ ) was added a $10 \times$ stock solution of $\mathrm{Zn}\left(\mathrm{NO}_{3}\right)_{2}\left(12 \mathrm{mM}\right.$ in $\left.\mathrm{H}_{2} \mathrm{O}, 10 \mu \mathrm{L}\right)$ or $\mathrm{H}_{2} \mathrm{O}(10 \mu \mathrm{L})$. The resulting solution was allowed to stand for $30 \mathrm{~min}$ at room temperature. After confirming gelation, a $10 \times$ stock solution of EDTA $(12 \mathrm{mM}, 50 \mathrm{mM}$ HEPES, $\mathrm{pH}$ $7.4,10 \mu \mathrm{L})$ or buffer $(10 \mu \mathrm{L})$ was added to the hydrogel, and incubated for $30 \mathrm{~min}$ at room temperature. The sample state (gel or sol) was confirmed by the tube inversion method. Photos were taken by iPhone 5 (Apple Inc.).

Glucose response of GOx-encapsulated $\mathrm{Zn}^{2+}$-induced hydrogels. To a freshlyprepared aqueous solution of BPmoc- $\mathrm{F}_{3}$ and GOx $(2.4 \mathrm{mM}$ and $1 \mathrm{mg} / \mathrm{mL}$, respectively, $50 \mathrm{mM}$ HEPES containing $0.5 \%$ glycerol, $\mathrm{pH} 7.4,100 \mu \mathrm{L}$ ) was added a $10 \times$ stock solution of $\mathrm{Zn}\left(\mathrm{NO}_{3}\right)_{2}\left(12 \mathrm{mM}, 0.5 \mathrm{eq}, 10 \mu \mathrm{L}\right.$ in $\left.\mathrm{H}_{2} \mathrm{O}\right)$. The resulting solution was incubated for $30 \mathrm{~min}$ at room temperature to form a transparent hydrogel. To the resultant hydrogel was added a $10 \times$ stock solution of glucose ( $48 \mathrm{mM}, 2.0 \mathrm{eq}, 10 \mu \mathrm{L}$ in $\mathrm{H}_{2} \mathrm{O}$ ), and then the resulting hydrogel was incubated at $30^{\circ} \mathrm{C}$ for $2 \mathrm{~h}$. The state (gel or sol) was determined by the tube inversion method. To determine the reaction rate by RP-HPLC, a solution of $p$-nitrobenzenesulfonamide $\left(1.6 \mathrm{mM}, 100 \mu \mathrm{L}\right.$ in $\left.\mathrm{CH}_{3} \mathrm{CN}\right)$ was added to the gel/sol, and the resultant solution was analyzed by RP-HPLC.

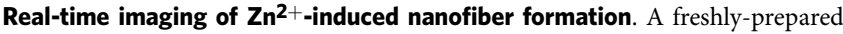
aqueous solution of BPmoc- $\mathrm{F}_{3}$ and BP-TMR $(1.6 \mathrm{mM}$ and $0.32 \mu \mathrm{M}$, respectively, $50 \mathrm{mM}$ HEPES, pH 7.4, $10 \mu \mathrm{L}$ ) was deposited on a Matsunami glass-bottom dish (non-coated, thickness: $0.16 \sim 0.19 \mathrm{~mm}$, catalog number: D11530H). A $10 \times$ stock solution of $\mathrm{Zn}\left(\mathrm{NO}_{3}\right)_{2}\left(8 \mathrm{mM}, 1.0 \mu \mathrm{L}\right.$ in $\left.\mathrm{H}_{2} \mathrm{O}\right)$ or $\mathrm{H}_{2} \mathrm{O}(1.0 \mu \mathrm{L})$ was added to the solution $1 \mathrm{~min}$ after starting time-lapse CLSM imaging. In Supplementary Fig. 8, a $10 \times$ stock solution of glucose $(32 \mathrm{mM}, 1 \mu \mathrm{L}), \mathrm{GOx}(10 \mathrm{mg} / \mathrm{mL}, 1 \mu \mathrm{L})$, or $\mathrm{H}_{2} \mathrm{O}(1 \mu \mathrm{L})$ was added $30 \mathrm{~min}$ before $\mathrm{Zn}\left(\mathrm{NO}_{3}\right)_{2}$ addition. The images and time-lapse movies of the $\mathrm{Zn}^{2+}$-induced formation of supramolecular nanofibers were obtained by LSM800 with the 100x objective. The overall fluorescent intensity of the field of view was calculated by Fiji.

Real-time imaging of glucose-responsive fiber degradation. A freshly-prepared aqueous solution of BPmoc- $\mathrm{F}_{3}, \mathrm{BP}-\mathrm{TMR}$, and GOx $(1.6 \mathrm{mM}, 0.32 \mu \mathrm{M}$ and $1.0 \mathrm{mg} /$ $\mathrm{mL}$, respectively, $50 \mathrm{mM}$ HEPES containing $0.5 \%$ glycerol, $\mathrm{pH} 7.4,11 \mu \mathrm{L}$ ) was deposited on a Matsunami glass-bottom dish. To this solution was added a 10× stock solution of $\mathrm{Zn}\left(\mathrm{NO}_{3}\right)_{2}\left(8 \mathrm{mM}, 1.0 \mu \mathrm{L}\right.$ in $\left.\mathrm{H}_{2} \mathrm{O}\right)$. The resulting solution was incubated for $30 \mathrm{~min}$ at room temperature. A $10 \times$ stock solution of glucose ( $32 \mathrm{mM}, 2.0 \mathrm{eq}, 1.0 \mu \mathrm{L}$ in $\mathrm{H}_{2} \mathrm{O}$ ) or $\mathrm{H}_{2} \mathrm{O}$ was added one minute after starting the time-lapse imaging. The time-lapse movies of nanofiber degradation were obtained by FV1000 with the $100 \times$ objective. The overall fluorescent intensity of the field of view was calculated by Fiji.

HPLC analysis of degradation kinetics of $\mathbf{B P m o c}-\mathbf{F}_{\mathbf{3}}$. To a freshly-prepared aqueous solution of BPmoc- $\mathrm{F}_{3}$ and GOx $(0.2 \mathrm{mM}$ and $1 \mathrm{mg} / \mathrm{mL}$, respectively, $50 \mathrm{mM}$ HEPES containing $0.5 \%$ glycerol, $\mathrm{pH} 7.4,110 \mu \mathrm{L}$ ) was added a solution of $\mathrm{Zn}\left(\mathrm{NO}_{3}\right)_{2}(8 \mathrm{mM}, 10 \mu \mathrm{L})$ or $\mathrm{H}_{2} \mathrm{O}(10 \mu \mathrm{L})$. To the resultant mixture was added a solution of glucose $(32 \mathrm{mM}, 10 \mu \mathrm{L})$, and then the resulting solution was incubated at $20^{\circ} \mathrm{C}$ for 15,30 , and $60 \mathrm{~min}$. To determine the reaction rate by RP-HPLC, a solution of $p$-nitrobenzenesulfonamide $\left(0.32 \mathrm{mM}, 100 \mu \mathrm{L}\right.$ in $\left.\mathrm{CH}_{3} \mathrm{CN}\right)$ was added to the reaction solution as an internal standard. The resultant solution was immediately analyzed by RP-HPLC.

Time-lapse CLSM imaging of the propagating wave. A freshly-prepared aqueous solution of BPmoc- $\mathrm{F}_{3}$, BP-TMR, and GOx $(1.6 \mathrm{mM}, 0.32 \mu \mathrm{M}$, and $1 \mathrm{mg} / \mathrm{mL}$, respectively, $50 \mathrm{mM}$ HEPES containing $0.5 \%$ glycerol, $\mathrm{pH} 7.4,5.5 \mu \mathrm{L})$ was deposited between a Matsunami micro cover glasses (bottom: non-coat, $30 \times 40 \mathrm{~mm}$, $0.12-0.17 \mathrm{~mm}$, top: $18 \times 18 \mathrm{~mm}, 0.12-0.17 \mathrm{~mm}$, non-coat. The cover glasses were sticked with four double-faced adhesive tape (Nichiban). A mixed solution of $\mathrm{Zn}$ $\left(\mathrm{NO}_{3}\right)_{2}$ and glucose $\left(4 \mathrm{mM}\right.$ and $16 \mathrm{mM}$, respectively, $1.0 \mu \mathrm{L}$ in $\left.\mathrm{H}_{2} \mathrm{O}\right)$ was added at the right edge of the droplet one minute after time-lapse imaging was started. The volumes of the droplet containing BPmoc- $\mathrm{F}_{3} / \mathrm{BP}-\mathrm{TMR} / \mathrm{GOx}$ and the solution of $\mathrm{Zn}$ $\left(\mathrm{NO}_{3}\right)_{2} /$ glucose were kept constant when applying different concentration of GOx and glucose. The time-lapse movies of a propagating wave were obtained by FV1000. In the case of CLSM imaging with the $100 \times$ objective, the images were acquired at $\sim 1 \mathrm{~mm}$ from the right edge of the droplet. In the case of the time-lapse movie acquired with the $100 \times$ objective, the fluorescent intensity of the ROI [size $(x \times y): 10$ pixels $\times 512$ pixels] at desired $x$ coordinates was calculated by Fiji. When using the $4 \times$ objective, fluorescent intensity of the ROI [size $(x \times y): 10$ pixels $\times 100$ pixels] at desired $x$ coordinates was calculated by Fiji.

CLSM imaging upon treatment of $\mathrm{Zn}\left(\mathrm{NO}_{3}\right)_{2}$ followed by glucose. The preparation of the droplet was the same as "time-lapse CLSM imaging of the propagating wave". A solution of $\mathrm{Zn}\left(\mathrm{NO}_{3}\right)_{2}\left(8 \mathrm{mM}, 0.5 \mu \mathrm{L}\right.$ in $\left.\mathrm{H}_{2} \mathrm{O}\right)$ was added to the right edge of the droplet. After $30 \mathrm{~min}$, a solution of glucose $(32 \mathrm{mM}, 0.5 \mu \mathrm{L}$ in $\mathrm{H}_{2} \mathrm{O}$ ) was added to the right edge of the droplet one minute after starting timelapse CLSM imaging. The images were acquired at $\sim 1 \mathrm{~mm}$ from the right edge of the droplet by FV1000.

CLSM imaging upon treatment of glucose followed by $\mathrm{Zn}\left(\mathrm{NO}_{3}\right)_{2}$. The preparation of the droplet was the same as "time-lapse CLSM imaging of the propagating wave". A solution of glucose $\left(32 \mathrm{mM}, 0.5 \mu \mathrm{L}\right.$ in $\left.\mathrm{H}_{2} \mathrm{O}\right)$ was added to the right edge of the droplet. After $5 \mathrm{~min}$, a solution of $\mathrm{Zn}\left(\mathrm{NO}_{3}\right)_{2}(8 \mathrm{mM}, 0.5 \mu \mathrm{L}$ in $\mathrm{H}_{2} \mathrm{O}$ ) was added to the right edge of the droplet one minute after starting timelapse CLSM imaging. The images were acquired at $\sim 1 \mathrm{~mm}$ from the right edge of the droplet by FV1000.

Preparation of Oregon Green-modified polystyrene beads. To a suspension of polystyrene beads (micromod, product code 01-01-502, diameter $500 \mathrm{~nm}, \mathrm{NH}_{2}$ modified, $50 \mathrm{mg} / \mathrm{mL}, 100 \mu \mathrm{L})$ were added $100 \mathrm{mM}$ HEPES buffer $(\mathrm{pH} 8.0,100 \mu \mathrm{L})$ PEG300-NHS (3.3 mg, $10 \mu \mathrm{mol}$, Quanta Biodesign), and Oregon Green 488-NHS $(0.3 \mu \mathrm{mol}, 6 \mu \mathrm{L}$ in DMSO, Thermo Fisher). The mixture was incubated at room temperature for $24 \mathrm{~h}$. The resultant mixture was centrifuged $\left(13500 \mathrm{rpm}, 4^{\circ} \mathrm{C}\right.$, $5 \mathrm{~min})$, the supernatant was removed, and then $\mathrm{H}_{2} \mathrm{O}(1 \mathrm{~mL})$ was added. This washing step was repeated 3 times. The washed beads were collected by centrifuge (13500 rpm, $\left.4^{\circ} \mathrm{C}, 5 \mathrm{~min}\right)$. To the resulting beads, $\mathrm{H}_{2} \mathrm{O}(100 \mu \mathrm{L})$ was added to obtain a suspension of Oregon Green-modified PEG-coated polystyrene beads.

In situ CLSM imaging of the bead displacement. The droplet containing BPmoc$\mathrm{F}_{3}$, BP-TMR, GOx, and Oregon Green-modified beads ( $1.6 \mathrm{mM}, 0.32 \mu \mathrm{M}, 1 \mathrm{mg} /$ $\mathrm{mL}$, and $20 \mu \mathrm{g} / \mathrm{mL}$, respectively, $5.5 \mu \mathrm{L}$ in $50 \mathrm{mM}$ HEPES, $\mathrm{pH}$ 7.4) were prepared by the same protocol as "time-lapse CLSM imaging of the propagating wave." A mixture of $\mathrm{Zn}\left(\mathrm{NO}_{3}\right)_{2}$ and glucose $\left(4 \mathrm{mM}\right.$ and $16 \mathrm{mM}$, respectively, $1.0 \mu \mathrm{L}$ in $\mathrm{H}_{2} \mathrm{O}$ ) was added to the droplet. The images were acquired by LSM800 with a 20x objective lens.

According to Stokes's law at low Reynold number, the force generated by the propagating wave was determined by the following equation.

$$
F_{d}=6 \pi \mu R \nu
$$

Here, $F_{\mathrm{d}}, \mu, R$, and $v$ are defined as the force applied to a spherical particle, viscosity of the solution, a radius and velocity of a spherical particle $(0.25 \mu \mathrm{m}$ and $0.4 \mu \mathrm{m} / \mathrm{s})$, respectively. We determined viscosity of a buffer $(50 \mathrm{mM}$ HEPES, $\mathrm{pH}$ 7.4), a solution of BPmoc- $\mathrm{F}_{3}$ before and after $\mathrm{Zn}\left(\mathrm{NO}_{3}\right)_{2}$ to be $1.1,1.3,2.5 \mathrm{mPa} \cdot \mathrm{s}$, respectively. Although the exact viscosity of the solution during the propagating 
wave could not be determined, we assumed that the maximum viscosity was lower than $2.5 \mathrm{mPa}$.s because the fluorescent intensity of the nanofibers in the propagating wave was lower than that in the mixture of BPmoc- $\mathrm{F}_{3}, \mathrm{BP}-\mathrm{TMR}$, and $\mathrm{Zn}\left(\mathrm{NO}_{3}\right)_{2}$. Therefore, the force generated by the propagating wave was calculated to be the order of $0.005 \mathrm{pN}$ with the viscosity value of $2.5 \mathrm{mPa} \cdot \mathrm{s}$.

Determination of the persistence length. To a solution of $\mathrm{BPmoc}-\mathrm{F}_{3}$ and BPTMR $(1.2 \mathrm{mM}, 0.34 \mu \mathrm{M}, 50 \mathrm{mM}$ HEPES ( $\mathrm{pH} 7.4), 50 \mu \mathrm{L})$, a solution of $\mathrm{Zn}\left(\mathrm{NO}_{3}\right)_{2}$ (8 mM, $5.0 \mu \mathrm{L}$ in $\mathrm{H}_{2} \mathrm{O}$ ) was added. After incubation at room temperature for $30 \mathrm{~min}$, the resultant viscous solution was vortexed for $30 \mathrm{~s}$ and transferred on a glass bottom dish. Time-lapse CLSM images were acquired at 33 frames/s by LSM800 with a $100 \times$ objective lens. Binary images were skeletonized and further spline interpolated using MATLAB to obtain sub-pixel fiber contour coordinates. The coordinates were used to calculate the 2-dimensional persistence length $L_{\mathrm{p}}$ (Supplementary Fig. 19a):

$$
\langle\cos [\Delta \theta(s)]\rangle=\exp \left(\frac{-s}{2 L_{p}}\right)
$$

where $\Delta \theta(s)$ is the angle change over the arc length.

\section{Data availability}

The authors declare that the data supporting the findings of this study are available with the paper and its Supplementary information files. The data that support the findings of this study are available from the corresponding author upon reasonable request.

Received: 31 March 2020; Accepted: 17 June 2020;

Published online: 15 July 2020

\section{References}

1. Whitesides, G. M. \& Grzybowski, B. Self-assembly at all scales. Science $\mathbf{2 9 5}$ 2418-2421 (2002).

2. Kholodenko, B. N. Cell-signalling dynamics in time and space. Nat. Rev. Mol. Cell Biol. 7, 165-176 (2006).

3. Novák, B. \& Tyson, J. J. Design principles of biochemical oscillators. Nat. Rev. Mol. Cell Biol. 9, 981-991 (2008).

4. Turing, A. M. The chemical basis of morphogenesis. Philos. Trans. R. Soc. Lond. B 237, 37-72 (1952).

5. Blanchoin, L., Boujemaa-Paterski, R., Sykes, C. \& Plastino, J. Actin dynamics, architecture, and mechanics in cell motility. Physiol. Rev. 94 235-263 (2014).

6. Krause, M. \& Gautreau, A. Steering cell migration: lamellipodium dynamics and the regulation of directional persistence. Nat. Rev. Mol. Cell Biol. 15, 577-590 (2014).

7. Soh, S., Byrska, M., Kandere-Grzybowska, K. \& Grzybowski, B. A. Reactiondiffusion systems in intracellular molecular transport and control. Angew. Chem. Int. Ed. 49, 4170-4198 (2010).

8. Neuman, K. C. \& Nagy, A. Single-molecule force spectroscopy: optical tweezers, magnetic tweezers and atomic force microscopy. Nat. Methods 5, 491-505 (2008).

9. Footer, M. J., Kerssemakers, J. W. J., Theriot, J. A. \& Dogterom, M. Direct measurement of force generation by actin filament polymerization using an optical trap. Proc. Natl Acad. Sci. Usa. 104, 2181-2186 (2007).

10. Marcy, Y., Prost, J., Carlier, M.-F. \& Sykes, C. Forces generated during actinbased propulsion: a direct measurement by micromanipulation. Proc. Natl Acad. Sci. Usa. 101, 5992-5997 (2004).

11. Kerssemakers, J. W. J. et al. Assembly dynamics of microtubules at molecular resolution. Nature 442, 709-712 (2006).

12. Grzybowski, B. A. \& Huck, W. T. S. The nanotechnology of life-inspired systems. Nat. Nanotechnol. 11, 585-592 (2016).

13. Epstein, I. R. \& Xu, B. Reaction-diffusion processes at the nano- and microscales. Nat. Nanotechnol. 11, 312-319 (2016).

14. Merindol, R. \& Walther, A. Materials learning from life: concepts for active, adaptive and autonomous molecular systems. Chem. Soc. Rev. 46, 5588-5619 (2017).

15. Boekhoven, J. et al. Dissipative self-assembly of a molecular gelator by using a chemical fuel. Angew. Chem. Int. Ed. 49, 4825-4828 (2010).

16. Carnall, J. M. A. et al. Mechanosensitive self-replication driven by selforganization. Science 327, 1502-1506 (2010).

17. Debnath, S., Roy, S. \& Ulijn, R. V. Peptide nanofibers with dynamic instability through nonequilibrium biocatalytic assembly. J. Am. Chem. Soc. 135, 16789-16792 (2013)

18. Dambenieks, A. K., Vu, P. H. Q. \& Fyles, T. M. Dissipative assembly of a membrane transport system. Chem. Sci. 5, 3396-3403 (2014).
19. Heuser, T., Steppert, A.-K., Lopez, C. M., Zhu, B. \& Walther, A. Generic concept to program the time domain of self-assemblies with a self-regulation mechanism. Nano Lett. 15, 2213-2219 (2015).

20. Pappas, C. G., Sasselli, I. R. \& Ulijn, R. V. Biocatalytic pathway selection in transient tripeptide nanostructures. Angew. Chem. Int. Ed. 54, 8119-8123 (2015).

21. Heuser, T., Weyandt, E. \& Walther, A. Biocatalytic feedback-driven temporal programming of self-regulating peptide hydrogels. Angew. Chem. Int. Ed. 54, 13258-13262 (2015).

22. Boekhoven, J., Hendriksen, W. E., Koper, G. J. M., Eelkema, R. \& van Esch., J. $\mathrm{H}$. Transient assembly of active materials fueled by a chemical reaction. Science 349, 1075-1079 (2015).

23. Wood, C. S., Browne, C., Wood, D. M. \& Nitschke, J. R. Fuel-controlled reassembly by metal-organic architectures. ACS Cent. Sci. 1, 504-509 (2015).

24. Maiti, S., Fortunati, I., Ferrante, C., Scrimin, P. \& Prins, L. J. Dissipative selfassembly of vesicular nanoreactors. Nat. Chem. 8, 725-731 (2016)

25. Lovrak, M. et al. Free-standing supramolecular hydrogel objects by reactiondiffusion. Nat. Commun. 8, 15317 (2017)

26. Tena-Solsona, M. et al. Non-equilibrium dissipative supramolecular materials with a tunable lifetime. Nat. Commun. 8, 15895 (2017).

27. Sorrenti, A., Leira-Iglesias, J., Sato, A. \& Hermans, T. M. Non-equilibrium steady states in supramolecular polymerization. Nat. Commun. 8, 15899 (2017).

28. Spitzer, D., Rodrigues, L. L., Straßburger, D., Mezger, M. \& Besenius, P. Tuneable transient thermogels mediated by a $\mathrm{pH}$ - and redox-regulated supramolecular polymerization. Angew. Chem. Int. Ed. 56, 15461-15465 (2017).

29. Sahoo, J. K., Pappas, C. G., Sasselli, I. R., Abul-Haija, Y. M. \& Ulijn, R. V. Biocatalytic self-assembly cascades. Angew. Chem. Int. Ed. 56, 6828-6832 (2017).

30. Jalani, K., Dhiman, S., Jain, A. \& George, S. J. Temporal switching of an amphiphilic self-assembly by a chemical fuel-driven conformational response. Chem. Sci. 8, 6030-6036 (2017).

31. Leira-Iglesias, J., Tassoni, A., Adachi, T., Stich, M. \& Hermans, T. M. Oscillations, travelling fronts and patterns in a supramolecular system. Nat. Nanotechnol. 13, 1021-1027 (2018).

32. Mishra, A. et al. Biomimetic temporal self-assembly via fuel-driven controlled supramolecular polymerization. Nat. Commun. 9, 1295 (2018).

33. Colomer, I., Morrow, S. M. \& Fletcher, S. P. A transient self-assembling selfreplicator. Nat. Commun. 9, 2239 (2018).

34. te Brinke, E. et al. Dissipative adaptation in driven self-assembly leading to self-dividing fibrils. Nat. Nanotechnol. 13, 849-855 (2018).

35. Jain, A., Dhiman, S., Dhayani, A., Vemula, P. K. \& George, S. J. Chemical fueldriven living and transient supramolecular polymerization. Nat. Commun. 10, 450 (2019).

36. Bal, S., Das, K., Ahmed, S. \& Das, D. Chemically fueled dissipative selfassembly that exploits cooperative catalysis. Angew. Chem. Int. Ed. 58, 244-247 (2019).

37. Singh, N., Lainer, B., Formon, G. J. M., De Piccoli, S. \& Hermans, T. M. Reprogramming hydrogel properties using a fuel-driven reaction cycle. J. Am. Chem. Soc. 142, 4083-4087 (2020).

38. Panja, S., Dietrich, B. \& Adams, D. J. Chemically fuelled self-regulating gel-togel transition. ChemSystemsChem 2, e1900038 (2020).

39. Yoshida, R., Takahashi, T., Yamaguchi, T. \& Ichijo, H. Self-oscillating gel. J. Am. Chem. Soc. 118, 5134-5135 (1996).

40. Maeda, S., Hara, Y., Sakai, T., Yoshida, R. \& Hashimoto, S. Self-walking gel. Adv. Mater. 19, 3480-3484 (2007).

41. Murase, Y., Maeda, S., Hashimoto, S. \& Yoshida, R. Design of a mass transport surface utilizing peristaltic motion of a self-oscillating gel. Langmuir $\mathbf{2 5}$, 483-489 (2009).

42. Lagzi, I., Kowalczyk, B., Wang, D. \& Grzybowski, B. A. Nanoparticle oscillations and fronts. Angew. Chem. Int. Ed. 49, 8616-8619 (2010).

43. Ikeda, M., Tanida, T., Yoshii, T. \& Hamachi, I. Rational molecular design of stimulus-responsive supramolecular hydrogels based on dipeptides. Adv. Mater. 23, 2819-2822 (2011).

44. Ikeda, M. et al. Installing logic-gate responses to a variety of biological substances in supramolecular hydrogel-enzyme hybrids. Nat. Chem. 6, 511-518 (2014).

45. Guascito, M. R., Malitesta, C., Mazzotta, E. \& Turco, A. Inhibitive determination of metal ions by an amperometric glucose oxidase biosensor: study of the effect of hydrogen peroxide decomposition. Sens. Actuators $B$ Chem. 131, 394-402 (2008).

46. Yamamoto, A. \& Ichikawa, M. Direct measurement of single soft lipid nanotubes: nanoscale information extracted in a noninvasive manner. Phys. Rev. E 86, 061905 (2012).

47. Gittes, F., Mickey, B., Nettleton, J. \& Howard, J. Flexural rigidity of microtubules and actin filaments measured from thermal fluctuations in shape. J. Cell Biol. 120, 923-934 (1993).

48. Freeman, R. et al. Reversible self-assembly of superstructured networks. Science 362, 808-813 (2018) 
49. Schindelin, J. et al. Fiji: an open-source platform for biological-image analysis. Nat. Methods 9, 676-682 (2012).

\section{Acknowledgements}

We thank S. Onogi (Kyoto Univ.) and H. Shigemitsu (Osaka Univ.) for their assistance of synthesis of BP-TMR. We thank Andrew Jackson, PhD, from Edanz Group (www. edanzediting.com/ac) for editing a draft of this manuscript. This work was supported by a Grant-in-Aid for Scientific Research on Innovative Areas "Chemistry for Multimolecular Crowding Biosystems” (JSPS KAKENHI Grant JP17H06348), JST ERATO Grant Number JPMJER1802 to I.H., and by a Grant-in-Aid for Young Scientists (JSPS KAKENHI Grant JP18K14333 and JP20K15400) to R.K.

\section{Author contributions}

I.H. and R.K. designed the project. R.K. conducted the gelation experiments and CLSM imaging. M.M. and M.I. performed numerical simulation and analysis. R.S. and M.T. analyzed the fluorescent beads displacement and the persistence length of supramolecular nanofibers. I.H. and R.K. wrote the manuscript and edited with all authors.

\section{Competing interests}

The authors declare no competing interests.

\section{Additional information}

Supplementary information is available for this paper at https://doi.org/10.1038/s41467020-17394-z.
Correspondence and requests for materials should be addressed to I.H.

Peer review information Nature Communications thanks Subi George and Rein V. Ulijn for their contribution to the peer review of this work. Peer reviewer reports are available.

Reprints and permission information is available at http://www.nature.com/reprints

Publisher's note Springer Nature remains neutral with regard to jurisdictional claims in published maps and institutional affiliations.

\section{(c) (1)}

Open Access This article is licensed under a Creative Commons Attribution 4.0 International License, which permits use, sharing, adaptation, distribution and reproduction in any medium or format, as long as you give appropriate credit to the original author(s) and the source, provide a link to the Creative Commons license, and indicate if changes were made. The images or other third party material in this article are included in the article's Creative Commons license, unless indicated otherwise in a credit line to the material. If material is not included in the article's Creative Commons license and your intended use is not permitted by statutory regulation or exceeds the permitted use, you will need to obtain permission directly from the copyright holder. To view a copy of this license, visit http://creativecommons.org/ licenses/by/4.0/.

(C) The Author(s) 2020 